\title{
Evidence for vocal performance constraints in a female nonhuman primate
}

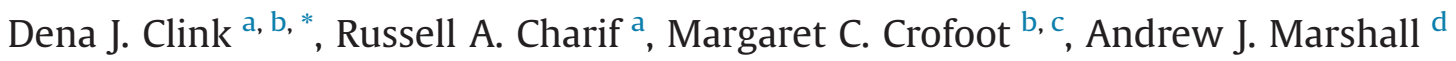 \\ a Bioacoustics Research Program, Cornell Laboratory of Ornithology, Cornell University, Ithaca, NY, U.S.A. \\ ${ }^{\mathrm{b}}$ Department of Anthropology, University of California, Davis, One Shields Avenue, Davis, CA, U.S.A. \\ c Smithsonian Tropical Research Institute, Balboa, Ancon, Panama \\ d Department of Anthropology, Program in the Environment, and School for Natural Resources and Environment, University of Michigan, Ann Arbor, MI, \\ U.S.A.
}

Keywords:

bandwidth

evolution of behaviour

great call

Hylobates

performance constraint

trade-off

trill

\begin{abstract}
Trilled vocalizations, wherein notes are repeated in rapid succession, are found in a variety of taxa including oscine birds, singing mice and nonhuman primates. Previous work on birds and singing mice has provided evidence of vocal performance constraints in trills, where there is a trade-off between the rate of the note repetition and the bandwidth (or frequency range) of each note. Here, we investigate vocal performance constraints in the trilled portion of the female contribution to the duet in the Bornean gibbon, Hylobates muelleri, recorded from seven sites in Sabah, Malaysia. We used two approaches. First, to ensure that our results were comparable with previous studies on vocal performance constraints, we used a $90 \%$ quantile regression to examine the relationship between trill rate and bandwidth. We found that there was a significant negative correlation between bandwidth and trill rate. Second, we formally compared multiple hierarchical models to identify the best predictors of bandwidth and trill rate. Our top model predicting bandwidth showed that trill rate and location within the trill were reliable predictors of bandwidth. With trill rate as the response variable, our top model included location within the trill as well as trill duration. We found that there were no important site-level differences in bandwidth but that trill rate varied predictably among sites. Our analyses provide strong evidence for performance constraints in the production of trills in Bornean gibbon females. Further research is needed to determine whether higher-performance trills provide honest signals of caller quality and whether gibbons respond differently to low- and high-performance calls.
\end{abstract}

Variation in acoustic signals is influenced by a wide range of factors including physical features of the environment, phylogenetic history of the organism and morphology and physiology of both the caller and the listener (Wilkins, Seddon, \& Safran, 2012). The laws of physics and structure of sound-producing organs further constrain the types of signals that animals can produce, and therefore the evolution of acoustic signals and communication systems (Fitch \& Hauser, 2003). Like all phenotypic traits, acoustic signals evolve through a combination of neutral and evolutionary mechanisms, and investigating the physical and mechanical limits on signal design can provide insight into the evolution of acoustic communication and diversity more broadly (Podos, Huber, et al.,

\footnotetext{
* Correspondence: D.J. Clink, Bioacoustics Research Program, Cornell Laboratory of Ornithology, Cornell University, 159 Sapsucker Woods Road, Ithaca, NY 14850, U.S.A.
}

E-mail address: djc426@cornell.edu (D. J. Clink).
2004). In addition, understanding the evolutionary constraints on the production of acoustic signals can provide insight into whether signals provide honest information about caller quality to the listener (e.g. Reby \& McComb, 2003).

A constraint on the production of acoustic signals that has garnered much interest is a 'vocal performance constraint' on trilled vocalizations where there is a trade-off between the rate of syllable repetition and the bandwidth (or range of frequencies) contained in each syllable (Podos, 1997). In birds and in one mouse species, there is documented evidence that this trade-off exists (Cramer \& Price, 2007; Cramer, 2013; Illes, Hall, \& Vehrencamp, 2006; Pasch, George, Campbell, \& Phelps, 2011; Podos, 1997; Podos, Huber, et al., 2004). Fast trills are predicted to be difficult to produce given the demands for rapid respiration and coordinated movements of the vocal tract (Podos, Huber, et al., 2004, Podos, Southall, \& Rossi-Santos, 2004). To produce trills at high bandwidths, animals must broadly and rapidly modulate their 
vocal tracts. Executing modulations of the vocal tract quickly and repetitively while maintaining the tonal frequencies of the vocalizations is physically challenging (Wilson, Bitton, Podos, \& Mennill, 2014). This biomechanical trade-off results in a pattern where frequency bandwidths at high trill rates are restricted and relatively narrow, but both narrow and wide bandwidths are possible at lower trill rates (Podos, 1997). Plots of note bandwidth versus trill rate exhibit a triangular distribution that reflects a performance trade-off in trill production, wherein the magnitude of frequency modulation (bandwidth) limits trill rate (or vice versa). The 'performance limit' of trills can be estimated by the upper limit of the regression of trill rate and bandwidth.

Understanding the evolutionary trade-offs in producing highperformance trills has been a topic of interest in the bird literature, with over 26 published studies reporting evidence of vocal performance constraints in birds (Wilson et al., 2014). Experiments on swamp sparrows, Melospiza georgiana, showed that hand-reared males that were exposed to artificially elevated trill rates were not able to reproduce trills at these elevated rates, instead producing songs with slower trill rates, omitted syllables or broken syntax (or pauses) between syllables (Podos, 1996). Songbirds learn natural model songs with a high degree of accuracy, so the inability to imitate the artificial models provides evidence of motor constraints on vocal performance. In birds, high fundamental frequencies have been shown to be accompanied by a wide beak opening or gape compared to that at lower fundamental frequencies (Podos, Huber, et al., 2004; Podos, Southall, et al., 2004), but in Neotropical singing mice, high-frequency sounds and gape width were not correlated, although singing mice did show evidence of performance constraints in trill vocalizations (Pasch et al., 2011). Other factors, such as respiratory musculature (Hartley \& Suthers, 1989) and motor control of the vocal tract (Riede, Suthers, Fletcher, \& Blevins, 2006) are also important in frequency modulation and rate of note production (Hartley \& Suthers, 1989), although the relative importance of these factors in shaping performance constraints are probably taxon specific.

Gibbons (Hylobatidae) are pair-living, territorial primates that occur throughout Southeast Asia. All of the approximately 20 species of gibbons produce species- and sex-specific vocalizations, and most species engage in coordinated duets with unique male and female components (Geissmann, 2002). In contrast to many bird species, the species-specific vocalizations are not learned, as shown in captive hybrid studies wherein hybrids produce calls that contain aspects from both parental species, despite only hearing the sex-specific call from one of the parental species (Brockelman \& Schilling, 1984; Geissmann, 1984). Female gibbons generally call along with their mothers as juveniles (Koda, Lemasson, Oyakawa, Rizaldi, Pamungkas, \& Masataka, 2013) or as adults in mated pairs (Brockelman \& Srikosamatara, 1993), with the exception of a few nonduetting gibbon species (e.g. Javan gibbons, Hylobates moloch: Ham, Hedwig, Lappan, \& Choe, 2016). The function of the female portion of the duet in gibbons appears to be related to territory defence, as females show a stronger response to playbacks at the centre of their territories (Mitani, 1985), and playbacks of female solos and duets often elicit female calling whether broadcast from the centre or boundary of their territory (Mitani, 1984). Unlike male birdsong, there is little evidence that the female contribution to the duet is the result of intersexual selection; rather, it appears to be the result of intrasexual competition among females.

Gibbons lack specialized amplifier organs such as the enlarged hyoidlaryngeal complex in howler monkeys (Schön, 1971), or the laryngeal airsac found in the siamangs (Riede, Tokuda, Munger, \& Thomson, 2008). Rather, gibbons produce their loud calls through dynamic control of their vocal tract, analogous to that of human soprano singers, where precise tuning of the supralaryngeal vocal tract results in the amplification of the fundamental frequency (Koda et al., 2012). This 'source-filter tuning' requires active control by the animal to produce loud vocalizations, and allows for gibbon calls to broadcast information to conspecifics in neighbouring territories over distances greater than $1 \mathrm{~km}$ (Mitani, 1985). In a few gibbon species, the female contribution to the duet contains trills, which are defined as vocalizations wherein frequency-modulated syllables are repeated in rapid succession (Podos, 1997). If the trade-off between trill rate and bandwidth is in fact the result of physical limitations on the production of broadband notes repeated in rapid succession, then this trade-off should be observed across taxa, regardless of taxonomic group or call function.

Here, we evaluate the evidence for vocal performance constraints in the trill portion of the great calls of Bornean gibbon, Hylobates muelleri, females. To our knowledge this study is one of the first to investigate performance constraints on female trills in any taxon, and one of the first to investigate performance constraints in vocalizations of a nonhuman primate (but see Terleph, Malaivijitnond, \& Reichard, 2016). Our study had two main objectives. First, we evaluated evidence for performance constraints in the trills of Bornean gibbon females using $90 \%$ quantile regression, so that our results are comparable to previous studies on vocal performance constraints (Wilson et al., 2014). And second, given the long duration of gibbon calls (up to 15 s; Clink, Bernard, Crofoot, \& Marshall, 2017) and the tendency of trill rate to increase over the course of a single call, we used multilevel models and model selection to determine which were the best predictor(s) of trill note bandwidth: trill rate $(\mathrm{Hz})$, placement within the trill sequence, placement of the call within the calling bout, or whether the calling bout was spontaneous or produced in response to artificially broadcast calls of conspecifics (playbacks). We also used multilevel models to test for the best predictor of trill rate and to investigate site-level variation in both bandwidth and trill rate.

\section{METHODS}

\section{Study Sites and Subjects}

We visited seven different sites across Sabah, Malaysia (Fig. 1, Table 1). Recordings were collected from January 2013 to August 2015 using a Marantz PMD 660 flash recorder equipped with a RODE NTG-2 directional condenser microphone. We recorded with a sample rate of $44.1 \mathrm{kHz}$ and a sample size of 16 bits and saved each file as a Waveform Audio (WAV) file. Our research focused on the female contribution to the duet, known as the great call (Geissmann, 2002). Bornean gibbon great calls consist of a combination of long (up to $1.8 \mathrm{~s}$ ), frequency-modulated notes as well as shorter, broadband trill notes, so we arbitrarily designated a cutoff duration of less than or equal to $0.135 \mathrm{~s}$ for trill notes. Calling bouts consist of alternating male and female contributions, and can last from a few minutes up to a few hours (D. J. Clink, personal observation), with a mean bout length of approximately 15 min (Mitani, 1985). Gibbon trills are distinct from most bird trills as they tend to be of a relatively long duration (3.5-15 s). Given the variable duration of gibbon trills and the typical gradual change in trill rate over the course of a trill, the fundamental unit of analysis for our study focuses on $1 \mathrm{~s}$ bins within trills that contain multiple trill notes (see Fig. 2).

Recording distances ranged from directly under the tree where the individual was calling to approximately $250 \mathrm{~m}$ away. In some cases, individuals moved over the course of a calling bout. Recording distance can influence bandwidth measures (Kroodsma, 2017), so we calculated the signal-to-noise ratio (a proxy for distance) for each $1 \mathrm{~s}$ bin used in our analysis; we only used $1 \mathrm{~s}$ bins where the signal-to-noise ratio was greater than $10 \mathrm{~dB}$ (see below 


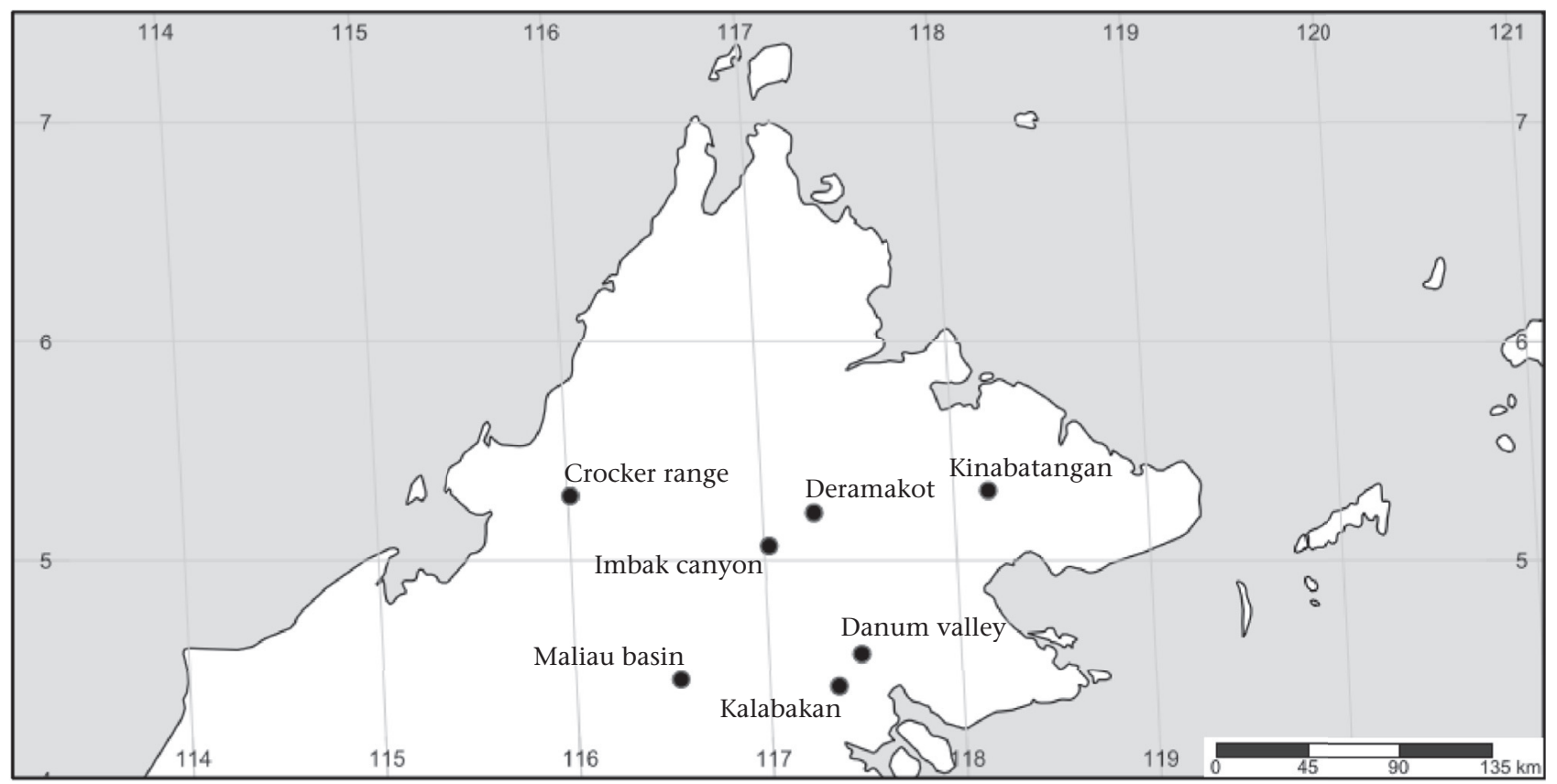

Figure 1. Map of sites visited in northern Borneo

Table 1

Site name, location, number of females, mean, standard deviation and range of calls from each female by site used for analysis

\begin{tabular}{|c|c|c|c|c|c|c|}
\hline Site & Latitude & Longitude & $\begin{array}{l}\text { Number } \\
\text { of females }\end{array}$ & $\begin{array}{l}\text { Number } \\
\text { of calls }\end{array}$ & $\begin{array}{l}\text { Mean (SD) of calls } \\
\text { per female }\end{array}$ & $\begin{array}{l}\text { Range of calls } \\
\text { per female }\end{array}$ \\
\hline Crocker Range National Park (CR) & 5.2934 & 116.01360 & 1 & 7 & 7 (NA) & $7-7$ \\
\hline Deramakot Forest Reserve (DK) & 5.2168 & 117.26544 & 9 & 107 & $11.9(6.7)$ & $2-25$ \\
\hline Danum Valley Conservation Area (DV) & 4.5752 & 117.47651 & 14 & 198 & $14.1(9.9)$ & $2-32$ \\
\hline Imbak Canyon Conservation Area (IC) & 5.0662 & 117.02557 & 9 & 153 & $17.0(14.1)$ & $5-42$ \\
\hline Kinabatangan Forest Reserve (KB) & 5.3178 & 118.17861 & 2 & 6 & $3.0(2.8)$ & $1-5$ \\
\hline Maliau Basin Conservation Area (MB) & 4.4528 & 116.53899 & 4 & 61 & $15.3(13.2)$ & $3-34$ \\
\hline $\begin{array}{l}\text { Stability of Altered Forest Ecosystems Project } \\
\text { in the Kalabakan Forest Reserve (SAF) }\end{array}$ & 4.4224 & 117.35560 & 24 & 314 & $13.1(8.5)$ & $3-43$ \\
\hline Total & & & 63 & 846 & $13.4(9.7)$ & $1-43$ \\
\hline
\end{tabular}

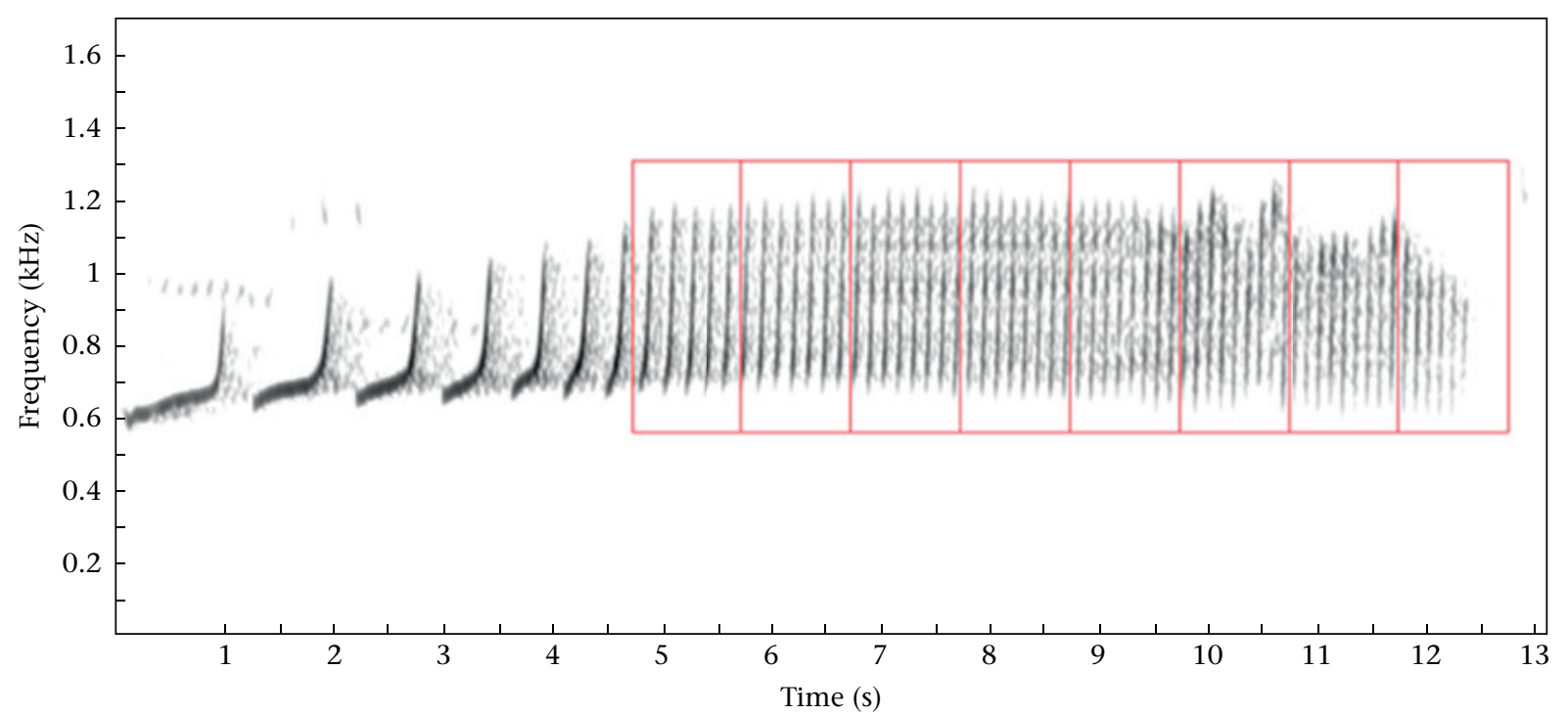

Figure 2. Spectrogram of Bornean gibbon female great call. The red boxes show the $1 \mathrm{~s}$ bins used for analysis; trill starts at the first note with duration of $0.135 \mathrm{~s}$ or lower. 
for details). What we consider to be an individual female for this analysis is one individual recording session at a particular location. In cases where we had recordings from the same location but taken during different days, we only used recordings from a single recording session to avoid including recordings from the same female on different days. Females that were recorded $>500 \mathrm{~m}$ apart (the approximate width of a gibbon territory; Brockelman \& Srikosamatara, 1993) were assumed to be different females. We also used group composition and unique behaviours (i.e. long calling bouts, unique male and/or female contribution to the duet, distinctive markings) as a means to distinguish between groups. Our study focused on wild, unhabituated gibbons, and therefore the length of territory tenure and ages of individuals were unknown. We analysed a total of 846 great calls from 63 gibbon females (mean $=13.4$ calls per female; range $1-43$ calls).

\section{Playback Protocol}

To augment data collection, we used playbacks to elicit calling by broadcasting a previously recorded duet approximately every $250 \mathrm{~m}$ along the established trail systems at each of our study sites. In the case that a group responded either by approaching the speaker or calling, we would not broadcast the playback again until approximately $500 \mathrm{~m}$ from the previous point, as this is the documented width of gibbon territories (Brockelman \& Srikosamatara, 1993). We used a Roland CUBE Street EX 4Channel 50 Watt Battery Powered Amplifier to broadcast a 3 min clip of a duet (at a standardized pressure of approximately $100 \mathrm{~dB}$ SPL at $1 \mathrm{~m}$ ) with a $30 \mathrm{~s}$ pause in between (and no more than five times total). During the pauses, we checked for vocal response and/ or physical approach to the speaker by gibbon individuals and immediately stopped the playbacks upon detection of gibbons. Most of the gibbon female calls ( 763 calls from 57 females) used in this analysis were collected in response to playbacks, with only 83 calls from six females collected during spontaneous calling bouts. To control for any confounding factors related to the quality of the call recording or features of the calls themselves, we used the same recording (collected at Maliau Basin Conservation Area, Sabah, Malaysia) where the female trill had a medium performance level (mean trill rate $=9.2$; mean bandwidth $=499 \mathrm{~Hz}$ ).

\section{Spectrogram Settings}

We used spectrograms to identify the presence of great calls within a recording, and also to identify the start and end time of notes within a great call. We created spectrograms using Raven Pro 1.5 sound analysis software (Cornell Lab of Ornithology Bioacoustics Research Program, Ithaca, NY, U.S.A.). Spectrograms were made with a 512-point ( $11.6 \mathrm{~ms}$ ) Hann window ( $3 \mathrm{~dB}$ bandwidth $=124$ $\mathrm{Hz}$ ), with $75 \%$ overlap and a 1024-point DFT, yielding time and frequency measurement precision of $2.9 \mathrm{~ms}$ and $43.1 \mathrm{~Hz}$. We used the band-limited energy detector in Raven to determine the start and end of trill notes (BLED configurations: minimum frequency: $0.40 \mathrm{kHz}$; maximum frequency: $1.60 \mathrm{kHz}$; minimum duration: 0.02 $\mathrm{s}$; maximum duration: $1.60 \mathrm{~s}$; minimum separation $0.04 \mathrm{~s}$ ), and DJC verified that each note was properly identified in the Raven selection tables.

\section{Signal-to-noise Ratio Calculation}

We calculated the signal-to-noise ratio for each $1 \mathrm{~s}$ bin in the $\mathrm{R}$ programming environment (R Development Core Team, 2017) using a custom function written by D.J.C. that relied heavily on functions in the package 'seewave' (Sueur, Aubin, \& Simonis, 2008). First, to estimate background noise of the recording, we extracted
$1 \mathrm{~min}$ before and $1 \mathrm{~min}$ after the start of each great call, under the assumption that there would be some portion of the $2 \mathrm{~min}$ recording that would contain only background noise (i.e. no gibbon calls or other vocalizing animals). Then, we filtered the files below $450 \mathrm{~Hz}$ and above $1800 \mathrm{~Hz}$ to focus on the gibbon frequency range. We then divided the $2 \mathrm{~min}$ recordings into $1 \mathrm{~s}$ bins (each containing 44100 samples). For each of these $1 \mathrm{~s}$ bins we further divided the bin into 100 time slices, each with a duration of $10 \mathrm{~ms}$ (441 samples). For each $10 \mathrm{~ms}$ slice, we summed the squares of the 441 samples, which provided the in-band power estimate for the $10 \mathrm{~ms}$ slice. We then took the median of the 10 ms slice powers for each 1 $\mathrm{s}$ bin as an estimate of the noise. This gave us 100 candidate noise estimates (one for each $1 \mathrm{~s}$ bin). To calculate a single noise power estimate for each gibbon great call, we used the 10th percentile of the distribution of the candidate noise estimates.

To calculate the power value for each $1 \mathrm{~s}$ bin of the gibbon call, we filtered the file as outlined above. We then divided the trill into $1 \mathrm{~s}$ bins, and further subdivided the bin into 100 time slices, each with a duration of $10 \mathrm{~ms}$ (441 samples) and summed the squares of the samples. We then had 100 candidate signal estimates for each 1 s trill bin, of which we used the 75th percentile of those values as our signal power estimate. The 75th percentile estimate still had noise mixed in, so we subtracted our estimated noise power from the power estimate. The signal-to-noise ratio, in decibels, was then computed as $\mathrm{SNR}_{\mathrm{dB}}=10 \times \log ($ signal/noise $)$.

We removed all $1 \mathrm{~s}$ bins with a signal-to-noise ratio of less than $10 \mathrm{~dB}$ from our analyses. $\mathrm{R}$ code for calculating signal-to-noise ratio is available as Supplementary material.

\section{Bandwidth and Trill Rate Calculation}

The use of spectrograms to estimate bandwidth can lead to under- or overestimation of bandwidth, depending on the amplitude of the signal, but the use of power spectra can circumvent many of these issues (Zollinger, Podos, Nemeth, Goller, \& Brumm, 2012). Therefore, we estimated bandwidth of each individual trill note using the power spectrum. We used the selection tables created in Raven to define the start and stop times of the trill notes. The power spectrum of the signal specifies the relative power as a function of frequency; it does not provide information on the change of signal over time. To calculate bandwidth, we first filtered the signal between 0.4 and $3.0 \mathrm{kHz}$ to prevent aliasing while downsampling. We then downsampled each .wav file to a sampling rate of $5 \mathrm{kHz}$, giving a Nyquist frequency of $2.5 \mathrm{kHz}$, which is well above the maximum frequency of Bornean gibbon trills $(\sim 1.8 \mathrm{kHz})$.

We downsampled so that we could obtain a frequency resolution of $1 \mathrm{~Hz}$ using a fast Fourier transform (FFT) size of 5000 samples, with a Hanning window function. If we did not downsample, we would have had to use an FFT size of 44100 samples (with a duration of $1 \mathrm{~s}$ ), which is much longer than the duration of our trill notes. Some trill notes were shorter in duration than 5000 samples, so to ensure that our samples were of sufficient length to compute the power spectrum we zero-padded them so that each trill note waveform had at least 5000 samples. To create a smoother curve for bandwidth estimation, we calculated a moving average over 100 frequency bins with an overlap of 50 frequency bins. Finally, to calculate the bandwidth, we measured the minimum and maximum frequency $-15 \mathrm{~dB}$ from the peak power of each spectrum (Fig. 3) and subtracted the minimum frequency from the maximum frequency to get bandwidth.

We calculated trill rate as the number of notes per second $(\mathrm{Hz})$, and averaged the bandwidth for all notes within each $1 \mathrm{~s}$ bin. In addition, over the course of a single great call, the amplitude of the trill tends to diminish from beginning to end, such that the terminal notes in the trill sometimes faded into the background noise, 


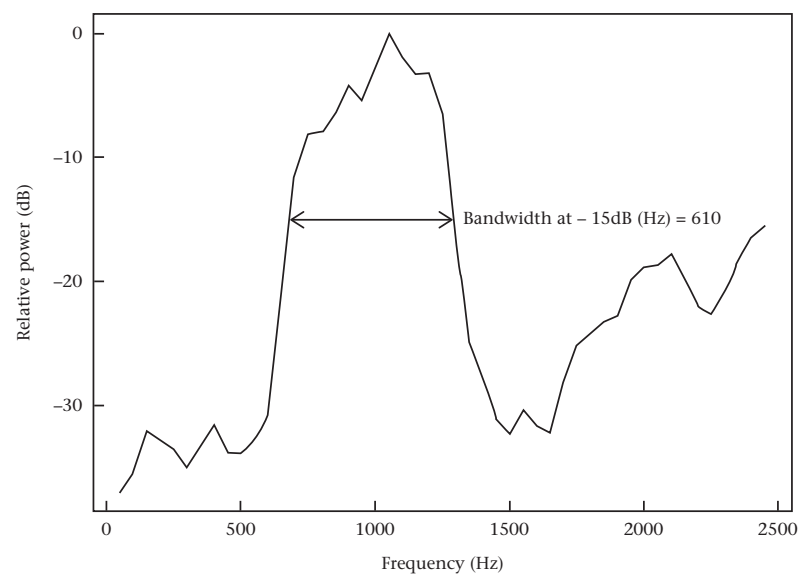

Figure 3. Representative power spectrum of a trill note used to measure bandwidth. The bandwidth measurement was performed at $-15 \mathrm{~dB}$ from the peak, and is indicated by the black line.

prohibiting reliable bandwidth measurements of those notes. Therefore, to ensure accurate estimates of bandwidth, we did not use the last $1 \mathrm{~s}$ bin from each great call. $\mathrm{R}$ code for calculating note bandwidth and trill rate is available as Supplementary material, and sound files are available at: https://github.com/DenaJGibbon/ Performance-Constraints.

To assess performance constraints, we first used quantile regression to investigate how changes in trill rate influence the 90th percentile of the bandwidth distribution (Wilson et al., 2014). We calculated the 90th percentile regression using the 'quantreg' package (Koenker, 2013). To meet the requirements of data independence for quantile regression, we calculated the average trill rate and bandwidth (over all the $1 \mathrm{~s}$ bins) for each female. Since taking the average for each female may result in the loss of important information, we also utilized hierarchical models to determine the best predictor of bandwidth. The use of hierarchical models allowed us to avoid averaging of data, calculate estimates using data with repeated and imbalanced sampling, and explicitly model variation within individuals, between individuals and between sites (McElreath, 2015).

First, we checked all predictor variables for multicollinearity (predictor variables summarized in Table 2) by calculating the variance inflation factor using the 'usdm' package (Naimi, 2015). After log transformation, bandwidth data were approximately normally distributed, so we used a Gaussian distribution. With

Table 2

Summary of predictor variables and random effects used in models

\begin{tabular}{ll}
\hline $\begin{array}{l}\text { Predictor variables } \\
\text { Bin }\end{array}$ & $\begin{array}{l}\text { Description } \\
\text { Location of } 1 \mathrm{~s} \text { bin containing trill notes within a single } \\
\text { great call (integer) } \\
\text { Binary variable indicating whether recordings were } \\
\text { collected during spontaneous duets or simulated } \\
\text { territorial intrusions }(0 \text { or } 1)\end{array}$ \\
Order of the great call in the calling bout (integer; \\
log transformed)
\end{tabular}

bandwidth as a response variable, we constructed a series of 12 hierarchical models (including the null model) that contained unique combinations of predictor variables (complete model list in Supplementary material Table S1) and compared these using the corrected Akaike's information criterion (AICc) and Akaike model weights. Each model reflects specific predictions (e.g. trill rate and location of $1 \mathrm{~s}$ bin containing trill notes within a single call (bin) will reliably predict bandwidth). Instead of providing $P$ values, the AICc model selection approach provides information about the magnitude of difference between models in their predictive power (Bolker et al., 2009).

For the models with trill rate as a response variable, we used a Poisson distribution, created a series of eight hierarchical models including the null model (complete model list in Supplementary material Table S2) and compared these models using AICc and Akaike model weights. Each model for trill rate and bandwidth included site, female and great call as nested random effects. We considered predictors reliable if the $95 \%$ confidence intervals of the beta coefficients did not overlap zero. We used the 'Ime4' and 'bbmle' packages (Bates, Mächler, Bolker, \& Walker, 2017; Bolker, 2014) to run the hierarchical models and calculate AICc and model weights.

We tested for the significance of between-site variance in bandwidth and trill rate by comparing two models for each response variable: the top model with site as a random effect, and the same model without site as a random effect (Pinheiro \& Bates, 2000). To further investigate site-level differences, we calculated the conditional modes using the 'ranef function in the 'Ime4' package, which calculates the difference between the predicted global average for a given response variable (bandwidth or trill rate), and the predicted response for a given site (Bates et al., 2017; Bolker et al., 2009). All statistical analyses were done in the $\mathrm{R}$ programming environment (R Development Core Team, 2017).

\section{Ethical Note}

All research was conducted in accordance with Malaysian laws and regulations, and with approval from the Sabah Biodiversity Council (access license number: JKM/MBS 1000-2/2 JLD.3 (42)) and in accordance with the University of California, Davis, Institutional Animal Care and Use Committee (IACUC) Protocol 29-30. Permission to conduct research in Crocker Range National Park was kindly provided by Sabah Parks. Permission to conduct research in Deramakot Forest Reserve was provided by the Sabah Forestry Department. Permission to conduct research in the Lower Kinabatangan Wildlife Sanctuary was granted by the Sabah Wildlife Department. Permission to conduct research in Danum Valley, Imbak Canyon and Maliau Basin was provided by their respective management committees and Yaysan Sabah. To minimize negative impacts on study subjects, the playback stimulus was played for no more than 15 min, playbacks were stopped immediately upon detection of focal group by the observer and playbacks were conducted within the presumed territory of a gibbon group no more than once per month.

\section{RESULTS}

\section{Quantile Regression Results}

In this study, we found strong evidence of performance constraints in the production of gibbon trills and substantial interindividual variation in bandwidth and trill rate. Using quantile regression, we found that there was a significant negative correlation between bandwidth and trill rate $(y=1097.86-64.57$, $P=0.01$; Figs. 4 and 5 ). 


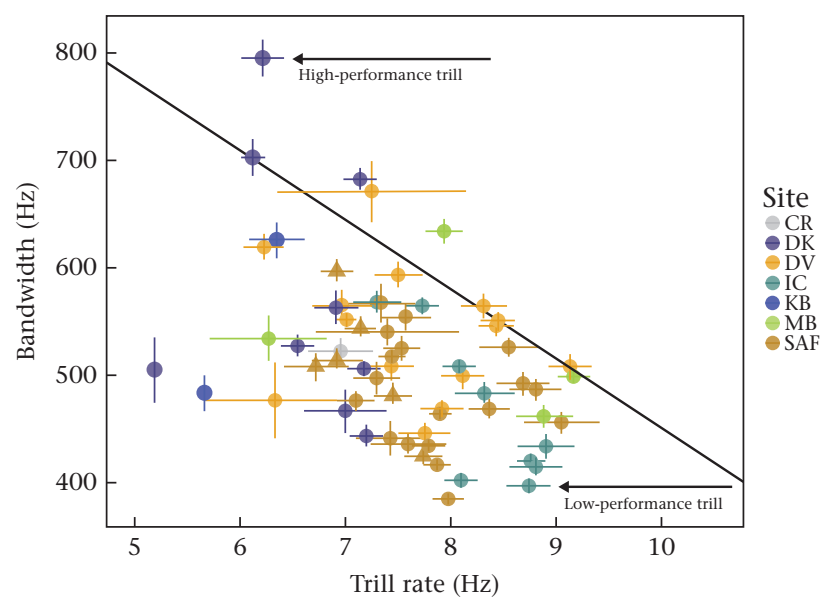

Figure 4. Evidence for the trade-off between note bandwidth and trill rate in Bornean gibbon female great calls. Each point represents the average value (calculated over $1 \mathrm{~s}$ bins) for one female, and the error bars are 95\% confidence intervals. Regression line was calculated using 90\% quantile regression. Upper left arrow indicates a female with a high-performance trill (representative spectrogram in Fig. 5a) and the lower right arrow indicates a female with a low-performance trill (representative spectrogram in Fig. 5b). Circles represent calls collected in response to playbacks, and triangles represent calls collected during spontaneous duetting. Different colour points represent females from different sites (see Table 1 for abbreviations).

\section{Model Selection Results}

Trill rate and bin (or location within the trill) were the most important predictors of bandwidth in the trills of Bornean gibbon female great calls, with models including trill rate and bin accounting for $>94 \%$ of the model weight (Table 3 ). Trill rate had a reliably negative effect on bandwidth (estimate -0.026 , $\mathrm{SE}=0.002$; Fig. 6), which means that as trill rate increased, bandwidth tended to decrease. Bin also had a reliably negative effect on bandwidth (estimate $=-0.025, \mathrm{SE}=0.002$; Fig. 6), which means that bandwidth tended to decrease over the course of a trill.
There were two reliable predictors of trill rate: bin and trill duration. The top model including bin and trill duration accounted for $<99 \%$ of the model weight (Table 3 ). Bin had a reliably positive effect on trill rate, which means that trill rate tended to increase over the course of a trill (estimate $=0.093, \mathrm{SE}=0.003$ ), whereas trill duration had a reliably negative effect, meaning that calls with longer trills tended to have slower trill rates (estimate $=-0.038$, $\mathrm{SE}=0.005$ ).

\section{Site-level Variation in Bandwidth and Trill Rate}

Including a site-level random effect only slightly improved the model fit for bandwidth, whereas including a site-level random effect substantially improved the model fit for trill rate (Table 4). All of the conditional modes for bandwidth had confidence intervals overlapping zero, providing further evidence that there were not important site-level differences in bandwidth, whereas there were important site-level differences in trill rate. Both Deramakot Forest Reserve (conditional mode $=-0.10, \mathrm{SE}=0.03$ ) and Imbak Canyon Conservation Area (conditional mode $=0.07, \mathrm{SE}=0.03$; Fig. 7) had trill rates that varied substantially from the predicted global mean trill rate.

\section{DISCUSSION}

We report the first documented evidence of performance constraints in the trill of a female nonhuman primate. To our knowledge, vocal performance constraints have not previously been documented in female vocalizations of any animal. Importantly, we provide evidence for vocal performance constraints in the trills of Bornean gibbon females, but we were not able to test whether trill performance provides information to conspecifics about the caller, or whether there is a link between trill performance and quality of the individual. Using quantile regression, we showed that there is a strong negative correlation between trill rate and bandwidth. Using a model selection framework, we showed that trill rate, but also location within the trill (bin), were important predictors of bandwidth in the trill notes of Bornean gibbon females. We found that

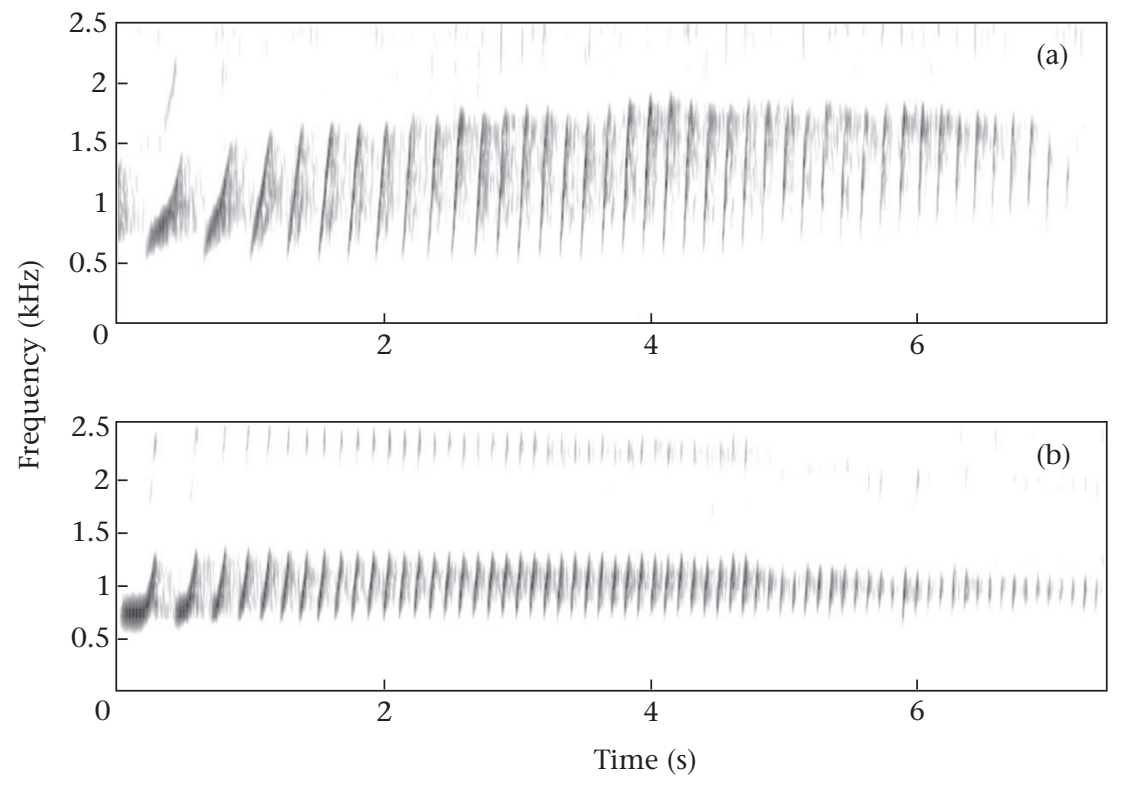

Figure 5. Representative spectrograms of (a) a high-performance trill (mean trill rate $=5.9$ notes $/ \mathrm{s}$, mean bandwidth $=674 \mathrm{~Hz}$ ) and $(\mathrm{b})$ a low-performance trill (mean trill rate $=8.8$ notes $/ \mathrm{s}$, mean bandwidth $=341 \mathrm{~Hz}$ ). High-performance trills occur close to (or above) the $90 \%$ quantile regression line, whereas low-performance trills are far below the regression line. Fig. 4 shows the mean performance of the two females illustrated here, relative to all others. 
Table 3

Akaike's information criterion (AICc) model comparison results for bandwidth and trill rate

\begin{tabular}{|c|c|c|c|c|c|c|}
\hline Model description & $d f$ & Log likelihood & $\Delta$ Log likelihood & AICc & $\Delta \mathrm{AICc}$ & AICc weight \\
\hline \multicolumn{7}{|l|}{ Bandwidth } \\
\hline Trill rate + bin & 7 & 2604.8 & 833.7 & -5195.6 & 0 & 0.82 \\
\hline Trill rate + bin + sequence & 8 & 2603.9 & 832.8 & -5191.8 & 3.9 & 0.12 \\
\hline Null model & 5 & 1772.9 & 1.7 & -3535.7 & 1659.9 & $<0.1$ \\
\hline \multicolumn{7}{|l|}{ Trill rate } \\
\hline Bin + trill duration & 6 & -8762.7 & 490.8 & 17537.4 & 0 & 0.99 \\
\hline Bin & 5 & -8781.7 & 471.8 & 18472.8 & 36.0 & $<0.1$ \\
\hline Null & 4 & -9253.5 & 0.0 & 18515.0 & 977.6 & $<0.1$ \\
\hline
\end{tabular}

For each response variable, the table shows the top two models and the null model. Each model included site, female and great call as nested random effects.

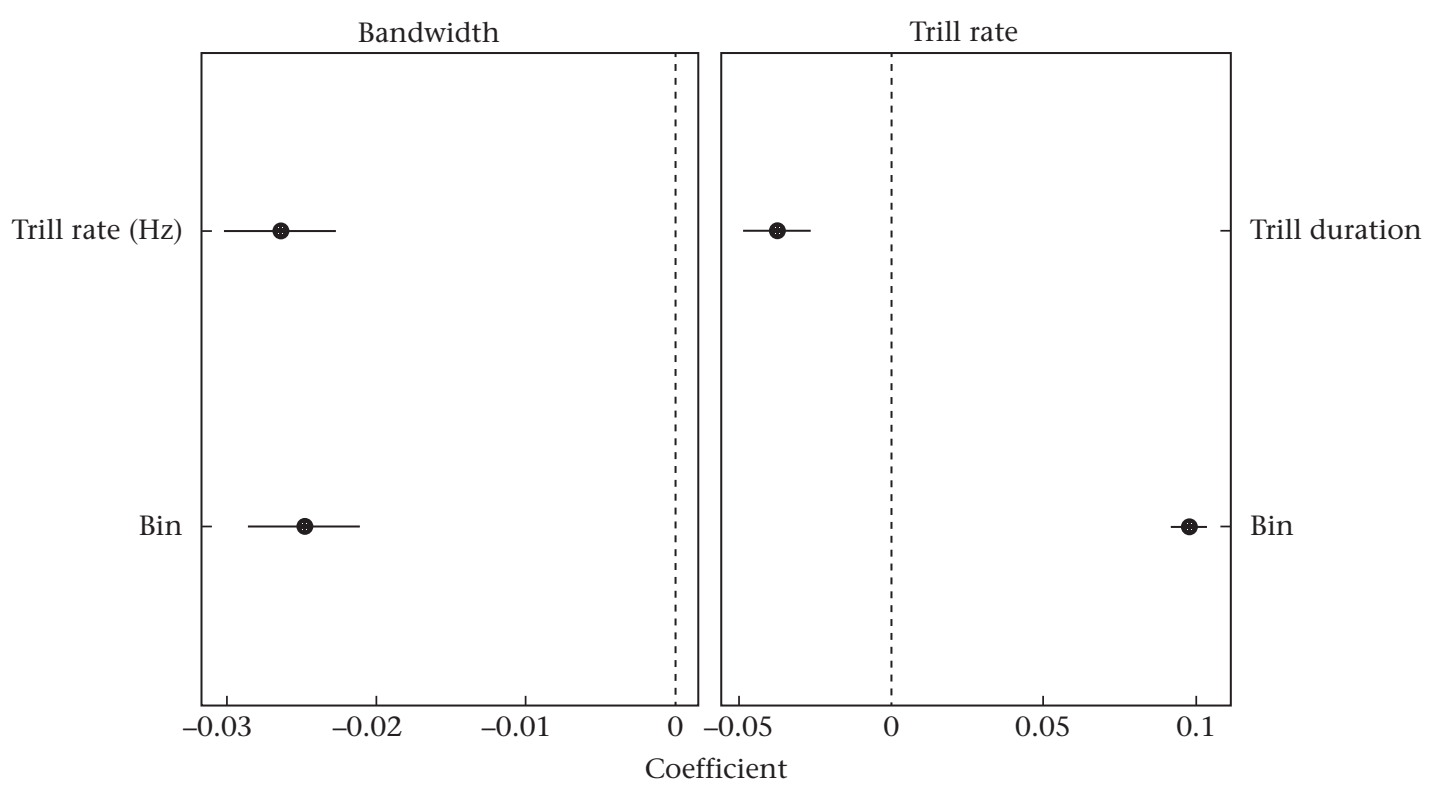

Figure 6. Coefficient estimates (along with 95\% confidence intervals) for top models of bandwidth and trill rate. Trill rate and bin were important predictors of bandwidth in Bornean gibbon trills, whereas bin and trill duration were important predictors of trill rate. Predictors were considered reliable if their $95 \%$ confidence intervals did not overlap zero.

there was indeed a trade-off between trill rate and bandwidth, and that bandwidth decreased over the course of the trill. We found no evidence that bandwidth or trill rate decrease over the course of a calling bout, or that simulated territorial intrusions influence gibbon trills. We also found that for trill rate both bin and trill duration were important predictors. Lastly, we found that there was important site-level variation in trill rate, but not bandwidth. We discuss the implications of our findings below.

\section{The 'Performance Hypothesis'}

There is substantial evidence for the existence of a trade-off between frequency bandwidth and rate of note repetition in birds (Ballentine, Hyman, \& Nowicki, 2004; Podos, 1996, 1997; and many other studies), and some evidence in mammals (Pasch et al., 2011; this study). The 'performance hypothesis' posits that it is difficult to produce trills at a faster rate and with wider frequency bandwidth; therefore, calls near the performance limits of trill rate and bandwidth may provide some indication of caller quality, with highperformance trills being performed by higher-quality individuals (Ballentine et al., 2004; Podos, 2016). For example, in the nightingale, Luscinia megarhynchos, the vocal performance of trills was found to predict age of the singing bird, with older males singing trills that are closer to the performance limit (Sprau, Roth, Amrhein, \& Naguib, 2013). In some bird species, females prefer trilled songs that are closer to the performance limits. For example, in the canary, Serinus canaria, females exhibited increased rates of copulation solicitation displays for broad-bandwidth vocalizations that

Table 4

Akaike's information criterion (AICc) model comparison results for top bandwidth and trill rate models with and without site as a random effect

\begin{tabular}{|c|c|c|c|c|c|c|}
\hline & $d f$ & Log likelihood & $\Delta$ Log likelihood & AICc & $\Delta$ AICC & AICc weight \\
\hline \multicolumn{7}{|l|}{ Bandwidth } \\
\hline Top model with site as random effect & 7 & 2618.9 & 1.1 & -5223.7 & 0 & 0.52 \\
\hline $\begin{array}{l}\text { Top model without site as random effect } \\
\text { Trill rate }\end{array}$ & \multicolumn{5}{|c|}{ Trill rate } & 0.48 \\
\hline Top model with site as random effect & 6 & -8762.7 & 4.6 & 17537.4 & 0 & 0.97 \\
\hline Top model without site as random effect & 5 & -8767.3 & 0 & 17544.6 & 7.3 & $<0.1$ \\
\hline
\end{tabular}

Models ranked by $\triangle$ AICc. Each model included female and great call as nested random effects. 

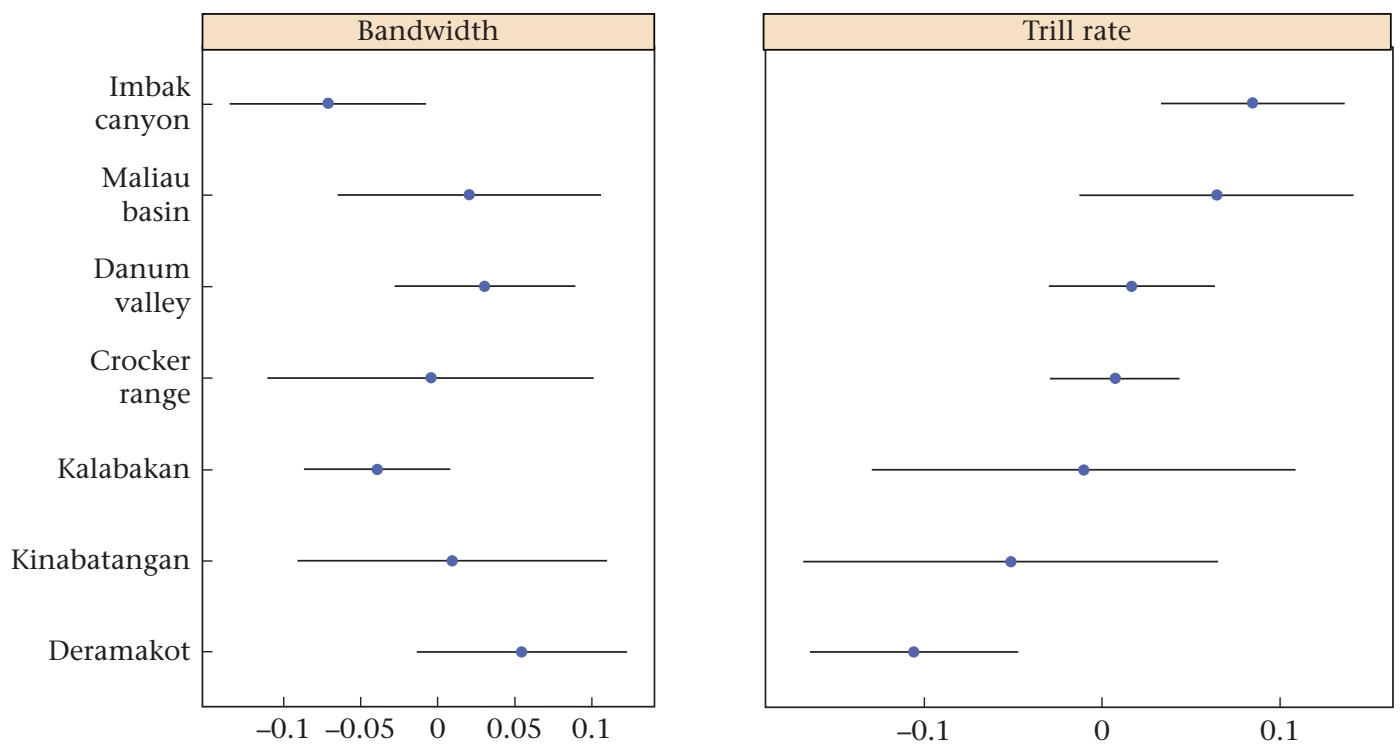

Figure 7. Site-level conditional modes for bandwidth and trill rate. Modes (dots) that are further from zero are less like the average response, and wider confidence intervals indicate greater variance for that particular site. We considered sites where the $95 \%$ confidence intervals do not overlap zero to have responses that differ significantly from the average response.

had artificially increased repetition rates (Drăgănoiu, Nagle, \& Kreutzer, 2002). A similar pattern was seen in swamp sparrows, $M$. georgiana, where females displayed significantly more to highperformance songs (Ballentine et al., 2004). But, this pattern is not universal, as in house wrens, Troglodytes aedon, trill performance was not correlated with male mating or reproductive success (Cramer, 2013).

Another potential measure of call performance is related to the animal's ability to perform each call or syllable with a high degree of consistency. For example, in a tropical mockingbird, Mimus gilvus, syllables of older males are more consistent, and consistent males tend to have higher dominance status and reproductive success (Botero et al., 2009). This is also the case for banded wrens, Thryothorus pleurostictus, where males exhibit higher levels of trill consistency as they get older (de Kort, Eldermire, Valderrama, Botero, \& Vehrencamp, 2009). Unlike most bird trills, note bandwidth in Bornean gibbon female trills tends to decrease over the course of a single call, making the trill structure within a single call inherently inconsistent, although some females do maintain relatively consistent bandwidth throughout the entire trill. Previously, we showed that there is little intraindividual variation in call features (Clink, Bernard, et al., 2017, Clink, Grote, Crofoot, \& Marshall, 2017), and in the present study, sequence of the call within a calling bout was not a reliable predictor of bandwidth or trill rate, providing further evidence for call consistency over time. Future research on Bornean gibbon females of known ages will be helpful in understanding whether there are age-related changes in trill rate or consistency.

A recent review critiqued many of the studies that reported support of the 'performance hypothesis' in birds, claiming that variation in trill performance is better explained by social factors and song learning as opposed to individual quality, and that trill rate depends mostly on when, where and from whom a bird learns its song, not the quality of the individual (Kroodsma, 2017). There have been numerous rebuttals to this critique that support the 'plausibility' of the performance hypothesis (Podos, 2016; Vehrencamp, de Kort, \& Illes, 2017). It is unclear whether any of this criticism is relevant to gibbons, as at present almost nothing is known about how gibbons acquire their calls, and whether there is a substantial learning component. In our study, we provide evidence that there is a trade-off between note bandwidth and trill rate, but we were not able to provide any test of the 'performance hypothesis' in Bornean gibbons. Previously, and corroborated by the current study, we found substantial interindividual variation in trill rate, and also important site-level differences, although the mechanisms that lead to this variation have yet to be elucidated (Clink, Grote, et al., 2017).

In birds, female territorial songs are associated with high levels of female-female competition (Langmore, 1998). Therefore, the strong contribution of the female gibbon to the duet may be indicative of a high degree of competition between female gibbons for either territories or mates (or both), and it seems plausible that at least some of the parameters of the great call, like the trill, provide indicators of female quality. For example, in the whitehanded gibbon, Hylobates lar, younger female great calls have greater bandwidth, higher fundamental frequency and shorter internote durations, and variation in the performance of whitehanded gibbon female calls may have evolved in the context of intrasexual selection to be an honest indicator of physical condition (Terleph et al., 2016). In male white-handed gibbons, higher levels of androgens are strongly correlated with higher fundamental frequencies (the opposite of the pattern observed in human vocalizations), providing more evidence that gibbon vocalizations may provide information regarding the signaller's quality (Barelli, Mundry, Heistermann, \& Hammerschmidt, 2013). In chimpanzees, Pan troglodytes, peak frequency of pant hoots, along with hourly and monthly rates of pant hooting, are positively correlated with testosterone levels, and pant hoots may provide information about a male's physical condition (Fedurek et al., 2016). Whether variation in the performance limits of gibbon trills is correlated with a female's physical condition, and whether variation in performance is detectable by other gibbons remains to be determined.

\section{Site-level Variation in Trills}

We show that there is important site-level variation in trill rate, but not in bandwidth of the trill notes, and this is supported by our previous analysis (Clink, Grote, et al., 2017). It has been proposed 
that habitat type can influence the presence of trills. For example, territorial oscine birds in open habitats tended to have notes with very short repetition periods in their songs (Wiley, 1991). Wiley proposed that a lack of notes with short repetition periods may help to reduce the effects of reverberation in forested environments. Wiley suggested that certain species in forested habitats may have retained the trills so that listeners can effectively judge the distance from the caller. It is also possible that site-level variation in vocalizations is nonadaptive, and the result of neutral evolutionary processes (Koerper \& Stickel, 1980). In pygmy marmosets, Cebuella pygmaea, there is evidence that degradation of the pulsatile structure may provide cues of caller distance to the listener (de la Torre \& Snowdon, 2002), and it is possible that gibbon trills also provide locational cues to the listener.

Interestingly, only four of the seven recognized gibbon species in the genus Hylobates exhibit trills ( $H$. muelleri; Hylobates klossi; $H$. moloch; Hylobates pileatus: Geissmann, 2002). Here we show that within a single gibbon species there are important differences between sites in trill rate, and it seems plausible that differences in forest structure may also influence trill rate (the acoustic adaptation hypothesis; Morton, 1975), but testing this hypothesis was beyond the scope of the present study. Although there has been limited support for the acoustic adaptation hypothesis in primates (Ey, Rahn, Hammerschmidt, \& Fischer, 2009; Hedwig, Mundry, Robbins, \& Boesch, 2015), the presence of site-level variation in trill rate warrants further research into the topic. It is also possible that site-level differences in trill rate are the result of population-level differences in age structure of females, as age has been shown to influence the structure of gibbons calls (Terleph et al., 2016).

\section{Future Directions Using Playback Experiments}

To our surprise, playbacks were not included in our top models as important predictors of either bandwidth or trill rate. One limitation in interpreting the importance of playbacks is that we used playbacks only as a means to elicit calling, and we did not conduct playback experiments, so we lacked data to compare calls produced before and after playback and calls produced during spontaneous duets and under simulated territorial intrusions. Birds have been shown to alter their vocal behaviour in response to simulated territorial intrusions, so it is possible that gibbons do as well. For example, in stripe-headed sparrows, Aimophila ruficauda, females were found to have a substantially increased song output during playbacks in comparison to baseline levels, and females sang $30 \%$ longer than males (Illes \& Yunes-Jimenez, 2009). This was also the case in purple-crowned fairy-wrens, Malurus coronatus, where simulated territorial intrusions lead to a substantial increase in pair song rate (Hall \& Peters, 2008). In Australian magpie-larks, Grallina cyanoleuca, simulated intrusions caused males and females to coordinate more of their vocalizations into duets (Hall, 2000). Indeed, during our data collection we noticed that groups tended to duet longer in response to simulated territorial intrusions, sometimes calling up to $4 \mathrm{~h}$ in response to playbacks, whereas typical spontaneous calling bouts last about 15 min (Mitani, 1985). Further studies using playback experiments are needed to determine whether gibbon females do in fact alter their calls in response to territorial intrusions. Playback experiments will also be useful to determine whether gibbons are able to detect differences in trills, and whether calls that are closer to performance limits elicit different behavioural responses from the listener.

\section{Conclusions}

We provide one of the first tests of vocal performance constraints in trill vocalizations produced by a nonhuman primate, although acoustic trade-offs have been documented previously in chimpanzee pant hoots (Fedurek, Zuberbühler, \& Semple, 2017) and gelada, Theropithecus gelada, vocal sequences (Gustison, Semple, Ferrer-i-Cancho, \& Bergman, 2016). We show that in Bornean gibbon females there is a trade-off between trill rate and trill note bandwidth, and also provide evidence that there is a trade-off between trill duration and trill rate. Interestingly, we did not find any relationship between note bandwidth and trill duration. The trade-offs between trill rate and bandwidth (or trill duration) provide strong evidence that trills are costly for individuals to produce. We also showed that there are important-site level differences in trill rate, but not bandwidth. Understanding the mechanisms that lead to interindividual variation, along with sitelevel variation, in the trill rate of Bornean gibbon female great calls may provide important insights into the function of these trills and what (if any) information they provide to conspecifics.

\section{Acknowledgments}

We thank Dr Henry Bernard, our Malaysian counterpart at the Biology Tropical and Conservation Institute, Universiti Malaysia Sabah for his support and collaboration. Funding to conduct this research was kindly provided by Primate Conservation, Inc., Cleveland Metroparks Zoo, the American Primatological Association and the Fulbright U.S. Student Program. We also gratefully acknowledge $\mathrm{Dr}$ Rob Ewers of Imperial College London for providing support for the data collection at the Stability of Altered Forest Ecosystems Project. We thank all of the staff at the Stability of Altered Forest Ecosystems project and the South East Asia Rainforest Research Programme (SEARRP) management for providing field and logistical support. We also thank members of the Crofoot lab (Brendan Barrett, Katrina Brock, Grace Davis, Allison Lau and Eric Van Cleave) for comments on earlier drafts of this manuscript. We also gratefully acknowledge the valuable input from the editor and two anonymous referees on earlier versions of this manuscript.

\section{References}

Ballentine, B., Hyman, J., \& Nowicki, S. (2004). Vocal performance influences female response to male bird song: An experimental test. Behavioral Ecology, 15(1), 163-168. https://doi.org/10.1093/beheco/arg090.

Barelli, C., Mundry, R., Heistermann, M., \& Hammerschmidt, K. (2013). Cues to androgens and quality in male gibbon songs. PLoS One, 8(12), e82748. https:// doi.org/10.1371/journal.pone.0082748.

Bates, D., Mächler, M., Bolker, B. M., \& Walker, S. (2017). lme4: Linear mixed-effects models using 'Eigen' and S4 ( $R$ package version 1.1-13). Vienna, Austria: R Foundation for Statistical Computing. https://cran.r-project.org/web/packages/ lme4/.

Bolker, B. M. (2014). bbmle: Tools for general maximum likelihood estimation (R Package Version 1.0.19). https://rdrr.io/cran/bbmle/.

Bolker, B. M., Brooks, M. E., Clark, C. J., Geange, S. W., Poulsen, J. R., Henry, M., et al. (2009). Generalized linear mixed models: A practical guide for ecology and evolution. Trends in Ecology \& Evolution, 24(3), 127-135. https://doi.org/10.1016/ j.tree.2008.10.008.

Botero, C. A., Rossman, R. J., Caro, L. M., Stenzler, L. M., Lovette, I. J., de Kort, S. R., et al. (2009). Syllable type consistency is related to age, social status, and reproductive success in the tropical mockingbird. Animal Behaviour, 77(3), 701-706. https://doi.org/10.1016/j.anbehav.2008.11.020.

Brockelman, W. Y., \& Schilling, D. (1984). Inheritance of stereotyped gibbon calls. Nature, 312(5995), 634-636. https://doi.org/10.1038/312634a0.

Brockelman, W. Y., \& Srikosamatara, S. (1993). Estimation of density of gibbon groups by use of loud songs. American Journal of Primatology, 29(2), 93-108. https://doi.org/10.1002/ajp.1350290203. 
Clink, D. J., Bernard, H., Crofoot, M. C., \& Marshall, A. J. (2017). Investigating individual vocal signatures and small-scale patterns of geographic variation in female Bornean gibbon (Hylobates muelleri) great calls. International Journal of Primatology, 38(4), 656-671. https://doi.org/10.1007/s10764-017-9972-y.

Clink, D. J., Grote, M. N., Crofoot, M. C., \& Marshall, A. J. (2017). A multivariate approach to investigating within-individual, between-individual and betweensite variation in Bornean gibbon female great calls. In Paper presented at the 4th International Symposium on Acoustic Communication by Animals Conference, July 2017, Omaha, NE.

Cramer, E. R. A. (2013). Physically challenging song traits, male quality, and reproductive success in house wrens. PLoS One, 8(3), 1-12. https://doi.org/10.1371/ journal.pone.0059208.

Cramer, E. R. A., \& Price, J. J. (2007). Red-winged blackbirds Ageliaus phoeniceus respond differently to song types with different performance levels. Journal of Avian Biology, 38(38), 122-127. https://doi.org/10.1111/j.2006.09088857.03839.x.

Drăgănoiu, T. I., Nagle, L., \& Kreutzer, M. (2002). Directional female preference for an exaggerated male trait in canary (Serinus canaria) song. Proceedings of the Royal Society B: Biological Sciences, 269(1509), 2525-2531. https://doi.org/10.1098 rspb.2002.2192.

Ey, E., Rahn, C., Hammerschmidt, K., \& Fischer, J. (2009). Wild female olive baboons adapt their grunt vocalizations to environmental conditions. Ethology, 115(5) 493-503. https://doi.org/10.1111/j.1439-0310.2009.01638.x.

Fedurek, P., Slocombe, K. E., Enigk, D. K., Emery Thompson, M., Wrangham, R. W., \& Muller, M. N. (2016). The relationship between testosterone and long-distance calling in wild male chimpanzees. Behavioral Ecology and Sociobiology, 70(5), 659-672. https://doi.org/10.1007/s00265-016-2087-1.

Fedurek, P., Zuberbühler, K., \& Semple, S. (2017). Trade-offs in the production of animal vocal sequences: Insights from the structure of wild chimpanzee pant hoots. Frontiers in Zoology, 14, 50.

Fitch, W., \& Hauser, M. (2003). Unpacking 'honesty': Vertebrate vocal production and the evolution of acoustic signals. In A. M. Simmons, A. N. Popper, \& R. R. Fay (Eds.), Acoustic communication (pp. 65-137). New York, NY: Springer-Verlag.

Geissmann, T. (1984). Inheritance of song parameters in the gibbon song, analysed in 2 hybrid gibbons (Hylobates pileatus $\times$ H. lar). Folia Primatologica, 42(3-4), 216-235. https://doi.org/10.1159/000156165.

Geissmann, T. (2002). Duet-splitting and the evolution of gibbon songs. Biological Reviews, 77(1), 57-76. https://doi.org/10.1017/S1464793101005826.

Gustison, M. L., Semple, S., Ferrer-i-Cancho, R., \& Bergman, T. J. (2016). Gelada voca sequences follow Menzerath's linguistic law. Proceedings of the National Academy of Sciences of the United States of America, 113(10), E2750-E2758. https:/ doi.org/10.1073/pnas.1522072113.

Hall, M. L. (2000). The function of duetting in magpie-larks: Conflict, cooperation, or commitment? Animal Behaviour, 60, 667-677. https://doi.org/10.1006 anbe.2000.1517.

Hall, M. L., \& Peters, A. (2008). Coordination between the sexes for territoria defence in a duetting fairy-wren. Animal Behaviour, 76, 65-73. https://doi.org 10.1016/j.anbehav.2008.01.010

Ham, S., Hedwig, D., Lappan, S., \& Choe, J. C. (2016). Song functions in nonduetting gibbons: Evidence from playback experiments on Javan gibbons (Hylobates moloch). International Journal of Primatology, 37, 225-240. https://doi.org 10.1007/s10764-016-9897-X.

Hartley, R. S., \& Suthers, R. A. (1989). Airflow and pressure during canary song: Direct evidence for mini-breaths. Journal of Comparative Physiology A, 165(1) 15-26. https://doi.org/10.1007/BF00613795.

Hedwig, D., Mundry, R., Robbins, M. M., \& Boesch, C. (2015). Audience effects, but not environmental influences, explain variation in gorilla close distance vocalizations: A test of the acoustic adaptation hypothesis. American Journal of Primatology, 77(12), 1239-1252. https://doi.org/10.1002/ajp.22462.

Illes, A. E., Hall, M. L., \& Vehrencamp, S. L. (2006). Vocal performance influences male receiver response in the banded wren. Proceedings of the Royal Society $B$ : Biological Sciences, 273(1596), 1907-1912. https://doi.org/10.1098/rspb. 2006.3535.

Illes, A. E., \& Yunes-Jimenez, L. (2009). A female songbird out-sings male conspecifics during simulated territorial intrusions. Proceedings of the Royal Society $B$ Biological Sciences, 276(1658), 981-986. https://doi.org/10.1098/rspb.2008. 1445 .

Koda, H., Lemasson, A., Oyakawa, C., Rizaldi, Pamungkas, J., \& Masataka, N. (2013). Possible role of mother-daughter vocal interactions on the development of species-specific song in gibbons. PLoS One, 8(8), e71432. https://doi.org/10.1371/ journal.pone.0071432.

Koda, H., Nishimura, T., Tokuda, I. T., Oyakawa, C., Nihonmatsu, T., \& Masataka, N. 2012). Soprano singing in gibbons. American Journal of Physical Anthropology. 149(3), 347-355. https://doi.org/10.1002/ajpa.22124.

Koenker, R. (2013). Quantreg: Quantile regression (R Package Version 5.33). https:// cran.r-project.org/web/packages/quantreg/.

Koerper, H. C., \& Stickel, E. G. (1980). Cultural drift: A primary process of culture change. Journal of Anthropological Research, 36(4), 463-469.

de Kort, S. R., Eldermire, E. R. B., Valderrama, S., Botero, C. A., \& Vehrencamp, S. L. (2009). Trill consistency is an age-related assessment signal in banded wrens.
Proceedings of the Royal Society B: Biological Sciences, 276(1665), 2315-2321. https://doi.org/10.1098/rspb.2009.0127.

Kroodsma, D. (2017). Birdsong performance studies: A contrary view. Animal Behaviour, 125, e1-e16. https://doi.org/10.1016/j.anbehav.2016.06.015.

Langmore, N. E. (1998). Functions of duet and solo songs of female birds. Trends in Ecology \& Evolution, 13(4), 136-140. https://doi.org/10.1016/S0169-5347(97) 01241-X.

McElreath, R. (2015). Statistical rethinking: A Bayesian course with examples in R and Stan. Boca Raton, FL: Chapman \& Hall/CRC.

Mitani, J. C. (1984). The behavioral regulation of monogamy in gibbons (Hylobates muelleri). Behavioral Ecology and Sociobiology, 15(3), 225-229. https://doi.org/ 10.1007/BF00292979.

Mitani, J. C. (1985). Gibbon song duets and intergroup spacing. Behaviour, 92(1/2), 59-96. https://doi.org/10.1080/0141192032000137321.

Morton, E. S. (1975). Ecological selection on avian sounds. American Naturalist. 109(965), 17-34.

Naimi, B. (2015). usdm: Uncertainty analysis for species distribution models (R package version 1.1-15). https://rdrr.io/cran/usdm/.

Pasch, B., George, A. S., Campbell, P., \& Phelps, S. M. (2011). Androgen-dependent male vocal performance influences female preference in Neotropical singing mice. Animal Behaviour, 82, 177-183. https://doi.org/10.1016/j.anbehav. 2011.04.018.

Pinheiro, J. C., \& Bates, D. M. (2000). Mixed-effects models in S and S-Plus. New York, NY: Springer-Verlag.

Podos, J. (1996). Motor constraints on vocal development in a songbird. Animal Behaviour, 51, 1061-1070. https://doi.org/10.1006/anbe.1996.0107.

Podos, J. (1997). A performance constraint on the evolution of trilled vocalizations in a songbird family (Passeriformes: Emberizidae). Evolution, 51(2), 537-551. https://doi.org/10.2307/2411126.

Podos, J. (2016). Birdsong performance studies: Reports of their death have been greatly exaggerated. Animal Behaviour, 125, e17-e24.

Podos, J., Huber, S. K., \& Taft, B. (2004). Bird song: The inferface of evolution and mechanism. Annual Review of Ecology, Evolution, and Systematics, 35, 55-87. https://doi.org/10.2307/annurev.ecolsys.35.021103.30000004.

Podos, J., Southall, J., \& Rossi-Santos, M. R. (2004). Vocal mechanics in Darwin's finches: Correlation of beak gape and song frequency. Journal of Experimental Biology, 207, 607-619. https://doi.org/10.1242/jeb.00770.

R Development Core Team. (2017). R: A language and environment for statistical computing. Vienna, Austria: R Foundation for Statistical Computing.

Reby, D., \& McComb, K. (2003). Anatomical constraints generate honesty: Acoustic cues to age and weight in the roars of red deer stags. Animal Behaviour, 65 519-530. https://doi.org/10.1006/anbe.2003.2078.

Riede, T., Suthers, R. A., Fletcher, N. H., \& Blevins, W. E. (2006). Songbirds tune their vocal tract to the fundamental frequency of their song. Proceedings of the $\mathrm{Na}$ tional Academy of Sciences of the United States of America, 103(14), 5543-5548. https://doi.org/10.1073/pnas.0601262103.

Riede, T., Tokuda, I. T., Munger, J. B., \& Thomson, S. L. (2008). Mammalian laryngseal air sacs add variability to the vocal tract impedance: Physical and computational modeling. Journal of the Acoustical Society of America, 124(1), 634-647. https://doi.org/10.1121/1.2924125.

Schön, M. A. (1971). The anatomy of the resonating mechanism in howling monkeys. Folia Primatologica, 15, 117-132. https://doi.org/10.1017/ CB09781107415324.004.

Sprau, P., Roth, T., Amrhein, V., \& Naguib, M. (2013). The predictive value of trill performance in a large repertoire songbird, the nightingale Luscinia megarhynchos. Journal of Avian Biology, 44(6), 567-574. https://doi.org/10.1111/ j.1600-048X.2013.00113.x.

Sueur, J., Aubin, T., \& Simonis, C. (2008). Seewave: A free modular tool for sound analysis and synthesis. Bioacoustics, 18, 213-226.

Terleph, T. A., Malaivijitnond, S., \& Reichard, U. H. (2016). Age related decline in female lar gibbon great call performance suggests that call features correlate with physical condition. BMC Evolutionary Biology, 16, 4. https://doi.org/10.1186/ s12862-015-0578-8.

de la Torre, S., \& Snowdon, C. T. (2002). Environmental correlates of voca communication of wild pygmy marmosets, Cebuella pygmaea. Animal Behaviour 63, 847-856. https://doi.org/10.1006/anbe.2001.1978.

Vehrencamp, S. L., de Kort, S. R., \& Illes, A. E. (2017). Response to Kroodsma's critique of banded wren song performance research. Animal Behaviour, 125 , e25-e28. https://doi.org/10.1016/j.anbehav.2016.12.004.

Wiley, R. (1991). Associations of Song properties with habitats for territorial oscine birds of eastern North America. American Naturalist, 138(4), 973-993.

Wilkins, M. R., Seddon, N., \& Safran, R. J. (2012). Evolutionary divergence in acoustic signals: Causes and consequences. Trends in Ecology \& Evolution, 28(3), 156-166. https://doi.org/10.1016/j.tree.2012.10.002.

Wilson, D. R., Bitton, P., Podos, J., \& Mennill, D. J. (2014). Uneven sampling and the analysis of vocal performance constraints. American Naturalist, 183(2), 214-228. https://doi.org/10.1086/674379.

Zollinger, S. A., Podos, J., Nemeth, E., Goller, F., \& Brumm, H. (2012). On the relationship between, and measurement of, amplitude and frequency in birdsong. Animal Behaviour, 84, e1-e9. https://doi.org/10.1016/j.anbehav.2012.04.026. 